\title{
Numerical Simulation and Vibration Analysis of Dynamically Loaded Bearing with Defect on Rolling Element
}

\author{
T. Govardhan and Achintya Choudhury \\ Sir Padampat Singhania University, Udaipur, India, 313601.
}

\author{
Deepak Paliwal \\ Madhav University, Abu road, India, 307026.
}

(Received 15 December 2015; accepted 26 April 2017)

The external loading on a rolling element bearing is often not of pure static nature but has a dynamic component associated with it. In the present study, the analysis of vibration response of rolling element bearing with localized defect on its rolling element subjected to external dynamic loading has been carried out. The dynamic component of external loading considered is either harmonic or random in nature. The defect-induced vibration signals have been processed with the FFT of their enveloped signal obtained from the Hilbert transform of band-passed signal around the resonant frequency band. Numerical results have been obtained for the NJ 204 bearing for different amplitude contributions of dynamic components of external load. For harmonic loading, the vibration spectra show additional spectral components through loading frequency as sidebands about each significant spectral components result due to static load. The experimental investigation confirms the presence of these additional components in the spectra. In the case of random loading, the noise level tends to rise with an increase in the value of standard deviation. The rise in noise level becomes so significant that some spectral components are masked by the noise for higher values of standard deviation.

\section{NOMENCLATURE}

A

$[\mathrm{A}]$

[B]

$b_{\text {def }}$

$[\mathbf{C}]$

$C_{0}$

$c$

$D$

[D]

$d$

$E$

$E^{\prime}$

$F_{r}$

$f$
$h_{f}$

$I$

K

$K_{i m p}$

$K_{0}$

$k \quad$ Stiffness of element

$L \quad$ Length of roller

$m \quad$ Mass of element

$n \quad \mathrm{n}^{\text {th }}$ revolution

$p_{d} \quad$ Diametral clearance of bearing

$P_{\max }(t)$

$P_{\psi}\left(t, F_{r}(t)\right)$

$R$

$R_{x, \text { race }}$
Amplitude of harmonic loading due to unbalanced mass

State matrix

Input matrix

Defect width

Dimensionless damping coefficient of fluid

film

Damping coefficient of element

Bearing pitch diameter

Direct transmission matrix

Diameter of roller

Modulus of elasticity

Effective modulus of elasticity

External radial loading on bearing

Linear frequency

Minimum film thickness

Moment of inertia of cross section of race

Load-deformation constant

Impact factor

Dimensionless stiffness coefficient of fluid

film

Maximum internal load on roller defect

Internal load distribution on roller defect

Radius of neutral axis

Effective radius of roller and race contact in

the direction of motion $t$

$u$

$u(t)$

$u_{r}(t)$

$W$

$x$

$y$

Z

$\beta$

$\psi_{l}(t)$

$\varepsilon(t)$

$\varepsilon_{o}$

$\omega$

$\omega_{n}$

$\eta_{o}$

$\xi$

$\lambda_{k}$

Subscripts:

$c \quad$ Cage

$s \quad$ Shaft

$f \quad$ Lubricating fluid

if Lubricant fluid for the conjunction of inner

race and roller

ir Inner race

of Lubricant fluid for the conjunction of outer race and roller

Outer race

$\begin{array}{ll}\text { or } & \text { Outer } \\ r & \text { Roller }\end{array}$

rd Roller defect

Superscripts:

$T$

Transpose 
Special symbols:

First derivative

Second derivative

\{\}

A vector

\section{INTRODUCTION}

Rolling element bearings are critical components of rotating machinery. It is therefore extremely important to detect the presence of defects in bearing elements because such defects cause the machinery to malfunction and may even lead to its catastrophic failure. Bearing elements are subjected to Hertzian stresses and are thus susceptible to fatigue failure. Micro-cracks are initially generated at the subsurface level and are propagated to the surface resulting in pits and spalls.

Vibration analysis has been used for bearing defect detection for the last four decades. Initial studies involved time domain analyses using peak value, r.m.s. value, and crest factor, as well as some statistical parameters such as probability density and kurtosis. Among these, kurtosis was found to be most effective for bearing defect detection. ${ }^{1}$ Early studies in frequency analysis involved identification of components at characteristic defect frequencies at which short-duration pulses were generated when a defect in one of the bearing elements strikes the mating surface. ${ }^{2}$ The expressions for these frequencies are listed in Table 1. ${ }^{1}$ Background noise due to macro-structure was a deterrent to defect detection from direct spectra. Many techniques were therefore applied to improve signal-to-noise ratio. The envelope detection method found the widest application among them. Resonances generated due to interaction of defects and mating elements are band-pass filtered, rectified, and subsequently low-pass filtered to identify the presence of characteristic defect frequencies to correlate them to the location of the defect. ${ }^{3}$ However, for non-stationary signals, this technique was found to be inadequate. ${ }^{4,5}$ Some time-frequency domain methodologies were, therefore, applied for bearing defect detection. Wavelet analysis has been found to be most effective among them. 6,7

For all the methods discussed so far, the load on the bearing was considered to be static in nature. However, rotating machinery is often subjected to dynamic loading. Kiral and Karagulle applied a finite element analysis-based model for detection of defects in bearings subjected to unbalanced loads. ${ }^{8}$ A multi-degree-of-freedom model was also proposed for bearings subjected to shock loading due to interaction of defect with the mating element. ${ }^{9}$ In this theoretical model, the effect of harmonic loading was also separately considered. Cong et al. proposed a model in which the bearing was subjected to a combination of static and harmonic loads for race defects. ${ }^{10}$ Authors made an investigation into the vibration response from bearings with defects on outer and inner races subjected to dynamic loading of harmonic and random nature. ${ }^{11}$

In the present work, a theoretical model has been proposed to obtain the vibration response of a bearing with a defect on one of the rolling elements. The load acting on the bearing has been considered to be a combination of static and dynamic loads. The dynamic component of the load may be either har-

Table 1. Characteristic defect frequencies for a rolling element bearing.

\begin{tabular}{|c|c|}
\hline Characteristic frequency & Expression \\
\hline Cage frequency, $f_{c}$ & $\left(f_{s} / 2\right)[1-(d / D) \cos \beta]$ \\
Outer race defect frequency, $f_{\text {od }}$ & $\left(Z f_{s} / 2\right)[1-(d / D) \cos \beta]$ \\
Inner race defect frequency, $f_{i d}$ & $\left(Z f_{s} / 2\right)[1+(d / D) \cos \beta]$ \\
Rolling element defect frequency, $f_{r d}$ & $\left(D f_{s} / d\right)\left[1-(d / D)^{2} \cos \beta\right]$ \\
\hline
\end{tabular}

monic or random in nature. Based on the proposed model, numerical results have been obtained and analysed. The theoretical results obtained for harmonic loading have been validated.

\section{NUMERICAL MODEL FOR VIBRATION RESPONSE}

The numerical model presented in this section includes excitations caused by roller defect, dynamic model of the bearing system, and identification of vibratory system parameters and their dynamics to obtain a vibration response of the system. The procedure of signal processing to obtain the amplitude of the demodulated envelope spectra has also been discussed in this section.

\subsection{Excitations Due to Roller Defect}

The strength of defect-induced excitations felt by bearing elements depends significantly on the bearing kinematic and dynamic parameters such as maximum normal approach between races along load line $\delta_{\text {max }}$, extent of symmetrical load zone about load line $\pm \psi_{l}$, load distribution factor $\varepsilon$, and maximum contact load along load line $P_{\max }$. These parameters can be viewed in Fig. 1.

The expressions for these parameters developed by Harris for the bearing under static loading have been suitably modified by the authors to ascertain the variations in these parameters under dynamic loading. ${ }^{12,14}$ From the numerical investigation, it was observed that all these parameters vary continuously with respect to time under dynamic loading but remain constant with respect to time in the case of static loading. The detailed investigations of these parameters under different kinds of loading have been carried out and presented in time and frequency domain. ${ }^{12}$

Sassi et al. suggested that the impulsive excitation produced by interaction of defect with the mating element would comprise two components: the load over the defect at the point of interaction in the load zone and the shock pulses generated due to the impact of the edges of the defect with the mating element. ${ }^{9}$ In order to determine the load over the defect in the load zone, the existence of non-stationary defect in the load zone can be found by comparing the values of instantaneous angular position of defect, $\omega_{c} t$ with that of load zones, $2\left(n_{c}-1\right) \pi+\psi_{l}$ and $2 n_{c} \pi-\psi_{l}$ for downward sense of loading when defect is executing its first and second half of each $n^{\text {th }}$ revolution, $n_{c}$ respectively, as shown in Fig. 1(a).

Whereas for upward sense of loading, the $\omega_{c} t$ has been compared with $\left(2 n_{c}-1\right) \pi-\psi_{l}$ and $2\left(n_{c}-1\right) \pi+\psi_{l}$ for first and second half of each $n^{\text {th }}$ revolution, $n_{c}$ respectively, as shown in Fig. 1(b). Therefore, the instances $t$ at which non-stationary defect appears in the load zone, which is the function of magnitude and direction of load on bearing as shown in Fig. 1, has been estimated with the help of the following expressions when defect is executing the first and second half of the revolutions, respectively, for different senses of load.

For downward sense of load:

$$
\left.\begin{array}{c}
\omega_{c} t \leq 2\left(n_{c}-1\right) \pi+\psi_{l}(t) \\
2 n_{c} \pi-\psi_{l}(t) \leq\left(\omega_{c} t\right) \leq 2 n_{c} \pi
\end{array}\right\}
$$

for upward sense of load:

$$
\left.\begin{array}{c}
\left(2 n_{c}-1\right) \pi-\psi_{l}(t) \leq\left(\omega_{c} t\right) \leq\left(2 n_{c}-1\right) \pi \\
\omega_{c} t \leq\left(2 n_{c}-1\right) \pi+\psi_{l}(t)
\end{array}\right\} ;
$$






(a)

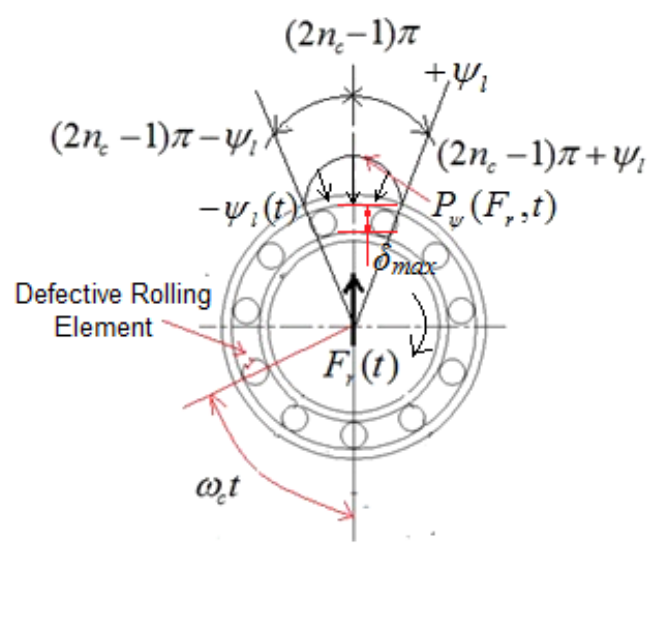

(b)

Figure 1. Geometrical locations of bearing kinematic and dynamic parameters: (a) downward sense of radial loading and (b) upward sense of radial loading.

where $\pm \psi_{l}(t)$ is the instantaneous extent of load zone.

The expression given by Harris to obtain load at the position of defect in a load zone for the case of static loading following Hertzian contact stress theory has been modified suitably to take the dynamic loading into account. ${ }^{13,14}$ The modified mathematical model to determine the load at the position of defect, $P_{\psi}$ with respect to the load line is a function of instantaneous external dynamic load and can be expressed as

$P_{\psi}\left(F_{r}, t\right)=\left\{\begin{array}{r}P_{\max }\left(F_{r}, t\right)\left[1-\frac{1}{2 \varepsilon\left(F_{r}, t\right)}(1-\cos \psi)\right]^{n} \\ \text { for }-\psi_{l}(t)<(\psi)<\psi_{l}(t) ; \\ 0 \quad \text { elsewhere. }\end{array}\right.$

In the above equation, $\psi$ depends on the location of defect and maximum contact load along the load line. $P_{\max }\left(F_{r}, t\right)$ can be expressed as ${ }^{14}$

$$
P_{\max }\left(F_{r}, t\right)=K \delta_{\max }^{n}(t)
$$

where $K$ is the load-deformation constant, for a steel roller bearing and ball bearing, $K$ can be expressed respectively as $^{14-16}$

$$
\begin{aligned}
& K=3.46 \times 10^{4} L^{8 / 9}\left(\mathrm{~N} / \mathrm{mm}^{1.11}\right) ; \\
& K=\frac{34300}{\kappa^{0.35}} d^{0.5}\left(\mathrm{~N} / \mathrm{mm}^{3 / 2}\right) ;
\end{aligned}
$$

where $\kappa=\frac{\beta_{o r}+\beta_{i r}-d}{d}$.

In the current study, because of the dynamic nature of the applied load, the load on defect at any angle is not constant with respect to time. Taking the varying nature of the load into account, the expression for excitation, $u_{r}$ as developed by Sassi et al. for ball bearing has been modified for cylindrical roller bearing as given in Eq. (3) ${ }^{9}$

$$
u_{r}\left(F_{r}, t\right)=P_{\psi}\left(F_{r}, t\right)\left[1+K_{i m p}\left(\frac{b_{d e f}}{d}\right)^{2}\right]
$$

where $b_{\text {def }}$ and $d$ are width of defect and diameter of the roller, respectively.

The excitations are generated at their respective characteristic defect frequencies, $f_{r d}$ shown in Table 1 when the defect comes in contact with its mating part in the load zone. The comprehensive numerical analysis of the modified mathematical model to estimate defect excitations generated in cylindrical roller bearing has been carried out in both time and frequency domains. ${ }^{13}$ In this analysis, the spectra of excitations are obtained for different amplitude ratios of static and dynamic parts of external load. The changes in spectra have been analysed for race and roller defects.

\subsection{Dynamic Model of Bearing System and System Parameters}

A 3 DOF vibratory model in the direction of maximum displacement proposed by Sassi et. al is considered in this work to determine the response of the dynamic system. The model has been presented in Fig. 2. The simplified model does not consider the effects of shaft, housing, and other supporting structures and focuses only on the flexural vibration of races. ${ }^{9}$ Flexure vibration of races has been considered in order to determine its stiffness. The natural frequency for the $j^{\text {th }}$ mode of flexural vibration of the races, $\omega_{n}$ can be obtained from the expression $^{17}$

$$
\omega_{n}=\frac{j\left(j^{2}-1\right)}{\sqrt{1+j^{2}}} \sqrt{\frac{E I}{\rho R^{4}}} .
$$

The stiffness of races, $k_{\text {race }}$ can be expressed as

$$
k_{\text {race }}=m_{\text {race }} \omega_{n}^{2} .
$$

In this work $k_{o r}$ and $k_{i r}$ have been obtained considering the elliptical mode of the races only to restrict the model to discrete one, i.e., $j=2$.

The stiffness and damping coefficients of the fluid film are given, respectively, and are modified to take the dynamic loading into account and can be expressed as ${ }^{18,19}$

$$
\begin{aligned}
& k_{f}=\frac{2 K_{0} F_{r}(t)}{p_{d}} ; \\
& c_{f}=\frac{2 C_{0} F_{r}(t)}{\omega p_{d}} ;
\end{aligned}
$$

were $p_{d}$ and $\omega$ are the diametral clearance and speed of bearing, respectively. $K_{0}$ and $C_{0}$ are, respectively, the dimensionless stiffness and damping coefficient of fluid film and can be 


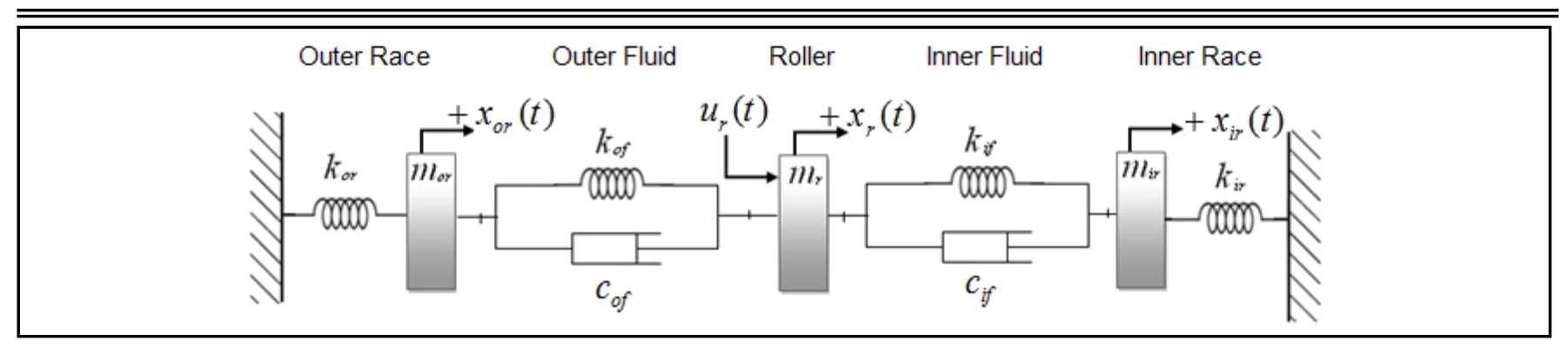

Figure 2. Equivalent vibratory model of bearing system.

$$
\begin{aligned}
& \text { written as }^{18,19}=\frac{4}{F_{r}(t) \lambda_{k}^{2}}\left[\frac{\varepsilon_{0}}{1-\varepsilon_{0}} \sin ^{2} \phi_{0}+\right. \\
& \left.\frac{3 \pi \varepsilon_{0}^{2}}{4\left(1-\varepsilon_{0}^{2}\right)^{5 / 2}} \sin \phi_{0} \cos \phi_{0}+\frac{2 \varepsilon_{0}\left(1+\varepsilon_{0}^{2}\right)}{\left(1-\varepsilon_{0}^{2}\right)^{3}} \cos ^{2} \phi_{0}\right] \\
& C_{0}=\frac{4}{F_{r}(t) \lambda_{k}^{2}}\left[\frac{\pi}{2\left(1-\varepsilon_{0}^{2}\right)^{3 / 2}} \sin ^{2} \phi_{0}+\right. \\
& \left.\frac{4 \varepsilon_{0}}{\left(1-\varepsilon_{0}^{2}\right)^{2}} \sin \phi_{0} \cos \phi_{0}+\frac{\pi\left(1+2 \varepsilon_{0}^{2}\right)}{2\left(1-\varepsilon_{0}^{2}\right)^{5 / 2}} \cos ^{2} \phi_{0}\right] \\
& \frac{4}{F_{r}(t) \lambda_{k}^{2}}=\frac{\left(1-\varepsilon_{0}\right)^{2}}{\varepsilon_{0}\left[16 \varepsilon_{0}+\pi^{2}\left(1-\varepsilon_{0}^{2}\right)\right]^{1 / 2}} \cos ^{2} \phi_{0} ; \\
& \tan \phi_{0}=\frac{\pi \sqrt{1-\varepsilon_{0}^{2}}}{4 \varepsilon_{0}} ; \\
& \varepsilon_{0}=1-\frac{h_{f}}{p_{d}} .
\end{aligned}
$$

The fluid thickness, $h_{f}$ has been computed using the following expression developed based on EHD theory ${ }^{20}$

$$
\begin{aligned}
h_{f}= & 3.63\left(\frac{\eta_{0} u}{E^{\prime} R_{x, \text { race }}}\right)^{0.68}\left(\xi E^{\prime}\right)^{0.49}\left(\frac{p_{\max }(t)}{E^{\prime} R_{x, \text { race }}^{2}}\right)^{-0.073} \\
& \left(1-e^{-0.68 k}\right) R_{x, \text { race }} ;
\end{aligned}
$$

where $k$ is the ellipticity parameter equal to infinity in case of line contact bearings. $k_{o f}$ and $k_{i f}$ can be obtained from Eq. (6) and $c_{o f}$ and $c_{i f}$ can be obtained from Eq. (7) by substituting the values of $K_{0}$ and $C_{0}$ with appropriate values of parameters for the fluid films concerned.

\subsection{Dynamics of Vibratory Model}

The equation of motion of each mass of the vibratory model of the bearing system shown in Fig. 3 can be derived by equating the resultant unbalanced force, due to stiffness, damping, and defect-induced excitation on each mass to its inertia force, $m \ddot{x}$. The set of forces under which each mass subjected to vibrations has been obtained from its free body diagram. The free body diagrams of outer race, rolling element, and inner race are shown in Fig. 3.

Therefore, the equation of motion of outer race, rolling element, and inner race, respectively, for the assumed positive displacement as shown in Fig. 3, $x(t)$ can be written as

$$
\begin{gathered}
-k_{o r} x_{o r}+k_{o f}\left(x_{r}-x_{o r}\right)+c_{o f}\left(\dot{x}_{r}-\dot{x}_{o r}\right)=m_{o r} \ddot{x}_{o r} ; \\
-k_{o f}\left(x_{r}-x_{o r}\right)-c_{o f}\left(\dot{x}_{r}-\dot{x}_{o r}\right)+k_{i f}\left(x_{i r}-x_{r}\right)+ \\
c_{i f}\left(\dot{x}_{i r}-\dot{x}_{r}\right)+u_{r}(t)=m_{r} \ddot{x}_{r} ; \\
-k_{i f}\left(x_{i r}-x_{r}\right)-c_{i f}\left(\dot{x}_{i r}-\dot{x}_{r}\right)-k_{i r} x_{i r}=m_{i r} \ddot{x}_{i r} .
\end{gathered}
$$

Equation (10a) is the set of second order and coupled differential equations representing the dynamics of vibration which are obtained by arranging Eq. (10) in a systematic manner:

$$
\begin{gathered}
m_{o r} \ddot{x}_{o r}+c_{o f} \dot{x}_{o r}-c_{o f} \dot{x}_{r}+\left(k_{o r}+k_{o f}\right) x_{o r}-k_{o f} x_{r}=0 ; \\
m_{r} \ddot{x}_{r}+c_{o f} \dot{x}_{o r}+\left(c_{o f}+c_{i f}\right) \dot{x}_{r}+c_{i f} \dot{x}_{i r}-k_{o f} x_{o r}+ \\
\left(k_{o f}+k_{i f}\right) x_{r}-k_{i f} x_{i r}=u_{r}\left(F_{r}, t\right) ; \\
m_{i r} \ddot{x}_{i r}-c_{i f} \dot{x}_{r}+c_{i f} \dot{x}_{i r}-k_{i f} x_{r}+\left(k_{i f}+k_{i r}\right) x_{i r}=0 .
\end{gathered}
$$

The response of the system will be obtained through the solution of differential equations of motion. The state space approach has been followed to solve the set of equations given by Eq. (10) because of its adaptability which provides scope to study single or multiple outputs against single and multiple inputs. With the help of this approach, the $n^{\text {th }}$ order differential equation can be written as first-order vector-matrix differential equations which are easily amenable to a solution using a digital computer.

Knowledge of a set of variables at initial time together with input excitations at or after initial time constitute the state variable vector, $\{y(t)\}$ and the input vector, $\{u(t)\}$ respectively, as expressed in Eqs. (11) and (12):

$$
\{y(t)\}=\left\{\begin{array}{llllll}
y_{1} & y_{2} & y_{3} & y_{4} & y_{5} & y_{6}
\end{array}\right\}^{T}
$$

where $y_{1}=x_{o r}, y_{2}=\dot{x}_{o r}, y_{3}=x_{r}, y_{4}=\dot{x}_{r}, y_{5}=x_{i r}$, and $y_{6}=\dot{x}_{i r}$

$$
\{u(t)\}=\left\{\begin{array}{lll}
0 & u_{r}(t) & 0
\end{array}\right\}^{T} .
$$

The state-space and output equations for the system under consideration can be expressed in terms of state and input excitation vector as:

$$
\{\dot{y}(t)\}=[\mathbf{A}]\{y(t)\}+[\mathbf{B}]\{u(t)\}
$$

and

$$
\{x(t)\}=[\mathbf{C}]\{y(t)\}+[\mathbf{D}]\{u(t)\}
$$

where

$$
\{x(t)\}=\left\{\begin{array}{lll}
x_{o r} & x_{r} & x_{i r}
\end{array}\right\}^{T}
$$

Analysing the set of differential equations, Eq. (10), to express in terms of state variables, the following expressions for the elements of $\{\dot{y}(t)\}$ may be obtained

$\dot{y}_{1}=y_{2}$ 


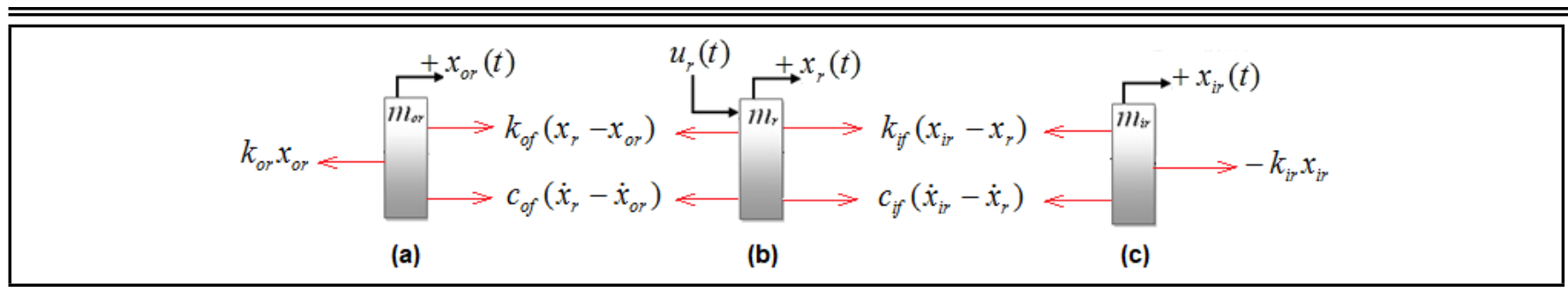

Figure 3. Free body diagram of mass of vibratory model: (a) outer race, (b) rolling element, and (c) inner race.

$$
\begin{aligned}
\dot{y}_{2}= & \ddot{x}_{o r}=-\frac{c_{o f} y_{2}}{m_{o r}}+\frac{c_{o f} y_{4}}{m_{o r}}-\frac{\left(k_{o r}+k_{o f}\right) y_{1}}{m_{o r}}+\frac{k_{o f} y_{3}}{m_{o r}} ; \\
\dot{y}_{3}= & y_{4} ; \\
\dot{y}_{4}= & \ddot{x}_{r}=\frac{u_{r}}{m_{r}}+\frac{c_{o f} y_{2}}{m_{r}}-\frac{\left(c_{o f}+c_{i f}\right) y_{4}}{m_{r}}+\frac{c_{i f} y_{6}}{m_{r}}+ \\
& \frac{k_{o f} y_{1}}{m_{r}}-\frac{\left(k_{o f}+k_{i f}\right) y_{3}}{m_{r}}+\frac{k_{i f} y_{5}}{m_{r}} ; \\
\dot{y}_{5}= & y_{6} ; \\
\dot{y}_{6}= & \ddot{x}_{i r}=\frac{c_{i f} y_{4}}{m_{i r}}-\frac{c_{i f} y_{6}}{m_{i r}}+\frac{k_{i f} y_{3}}{m_{i r}}-\frac{\left(k_{i f}+k_{i r}\right) y_{5}}{m_{i r}} .
\end{aligned}
$$

The matrices $[\mathbf{A}],[\mathbf{B}],[\mathbf{C}]$, and $[\mathbf{D}]$ can be obtained by rearranging the Eqs. (14)-(14e) in the form of Eqs. (13a) and (13b) and may be expressed as follows:

$$
\begin{aligned}
& {[\mathbf{A}]=} \\
& {\left[\begin{array}{cccccc}
0 & 1 & 0 & 0 & 0 & 0 \\
-\frac{k_{o r}+k_{o f}}{m_{o r}} & -\frac{c_{o f}}{m_{o r}} & \frac{k_{o f}}{m_{o r}} & \frac{c_{o f}}{m_{o r}} & 0 & 0 \\
0 & 0 & 0 & 1 & 0 & 0 \\
\frac{k_{o f}}{m_{r}} & \frac{c_{o f}}{m_{r}} & -\frac{k_{o f}+k_{i f}}{m_{r}} & -\frac{c_{o f}+c_{i f}}{m_{r}} & \frac{k_{i f}}{m_{r}} & \frac{c_{i f}}{m_{r}} \\
0 & 0 & 0 & 0 & 0 & 1 \\
0 & 0 & \frac{k_{i f}}{m_{i r}} & \frac{c_{i f}}{m_{i r}} & -\frac{k_{i f}+k_{i r}}{m_{i r}} & -\frac{c_{i f}}{m_{i r}}
\end{array}\right]}
\end{aligned}
$$

$$
\begin{gathered}
{[\mathbf{B}]=\left[\begin{array}{lll}
0 & 0 & 0 \\
0 & 0 & 0 \\
0 & 0 & 0 \\
0 & 0 & 0 \\
0 & 0 & 0 \\
0 & 0 & 0
\end{array}\right] ;} \\
{[\mathbf{C}]=\left[\begin{array}{llllll}
1 & 0 & 0 & 0 & 0 & 0
\end{array}\right] ;}
\end{gathered}
$$

and $[\mathbf{D}]$ is a null matrix.

\subsection{Simulation and Fault Diagnosis}

In the current study, the velocity response of outer race has been further processed to obtain bearing fault diagnostic information. It is not easy to detect smaller diagnostic frequencies by conventional spectral analysis in the presence of greater resonant frequencies of the vibratory system and noise from other sources. It is usual to consider the resonance as being amplitude modulated at characteristic defect frequency. The defect diagnostic information, therefore, will be in the frequency band around the system resonant frequency, so the response signal is band pass filtered in the high frequency band around the system resonant frequency. The filtered signal in time domain is then amplitude demodulated to form the envelope.
Table 2. Geometry of NJ 204 bearing.

\begin{tabular}{|ll|ll||}
\hline Bore & $: 20 \mathrm{~mm}$ & Diametral clearance & $: 50 \mu \mathrm{m}$ \\
Width & $: 14 \mathrm{~mm}$ & Contact angle & $: 0^{\circ}$ \\
Pitch diameter & $: 34 \mathrm{~mm}$ & Diameter of each roller $: 7.5 \mathrm{~mm}$ \\
Outside diameter & $: 47 \mathrm{~mm}$ & No. of rollers & $: 11$ \\
Length of each roller $: 9 \mathrm{~mm}$ & Mass of outer race & $: 0.05715 \mathrm{~kg}$ \\
Mass of roller & $: 0.00315 \mathrm{~kg}$ & Mass of inner race & $: 0.027 \mathrm{~kg}$ \\
\hline
\end{tabular}

Hilbert Transform is applied to obtain the envelope of the signal whose spectrum contains the desired diagnostic information in terms of repetition of characteristic defect frequency as well as modulation by appropriate frequency.

\section{NUMERICAL COMPUTATION AND DISCUSSION OF RESULTS}

The mathematical formulations discussed in earlier sections and numerical procedure explained in Fig. 4 are applied to an NJ 204 cylindrical roller bearing whose geometrical parameters are specified in Table 2 with $500 \mu \mathrm{m}$ roller defect width to obtain numerical results for different types of dynamic loading. The bearing is assumed to be rotating at a shaft speed of $1000 \mathrm{rpm}$. At this speed the values of cage, shaft, and roller defect frequencies are $6.49 \mathrm{~Hz}, 16.67 \mathrm{~Hz}$, and $71.88 \mathrm{~Hz}$, respectively. The load considered in the present work has been assumed to have a static component of $1000 \mathrm{~N}$ along with a variable component of harmonic or random nature. The harmonic and random loading have been varied to investigate the effects of dynamic loading on the resultant spectra and the findings have been discussed in detail in the following subsections.

\subsection{External Harmonic Loading}

The external dynamic load on bearing has been considered to be a combination of static load, $W$ and harmonic load of amplitude, $A$. The harmonic load has been assumed to be due to unbalance of shaft and is thus associated with the shaft frequency, $f_{s}$. The external radial load, therefore, can be expressed as follows:

$$
F_{r}=W+A \cos \left(2 \pi f_{s} t\right)
$$

The different bearing dynamic parameters, viz., internal load distribution over the defect and load zone which in turn depend on various bearing kinematic parameters, viz., max. contact deflection along load line and load distribution factor has been considered as time varying in the present study. Instantaneous variations in these parameters have been depicted in Fig. 5 for a bearing running at $1000 \mathrm{rpm}$ and subjected to a load having a static component of $W=1000 \mathrm{~N}$ and a harmonic component of amplitude $A=500 \mathrm{~N}$. It can be observed that all these parameters will continuously change with time for the given load condition. For static load, all these parameters remain constant with respect to time. ${ }^{11}$

The non-periodic load distribution and excitations are calculated and input to the state space model in order to determine 


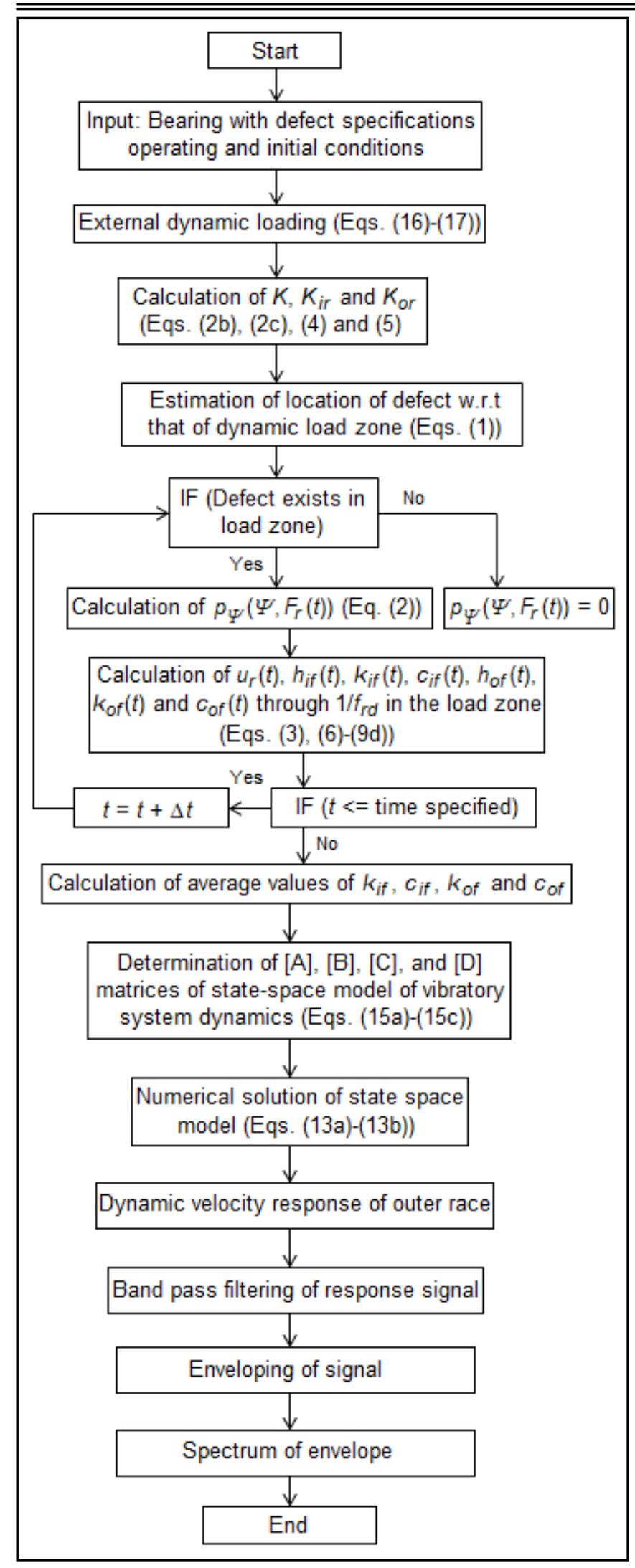

Figure 4. Flow chart of numerical procedure.

response of outer race, $x_{\text {or }}$ of the bearing due to roller defect. The response is shown in Fig. 6(a). From the Fig. 6(b) the resonant frequency of the vibratory system can be found to be at $37.37 \mathrm{kHz}$. The time domain signal, therefore, has been filtered through a frequency band of 35 to $40 \mathrm{kHz}$ and the band pass filtered signal is then demodulated using Hilbert transform as shown in Figs. 6(c) and 6(d).

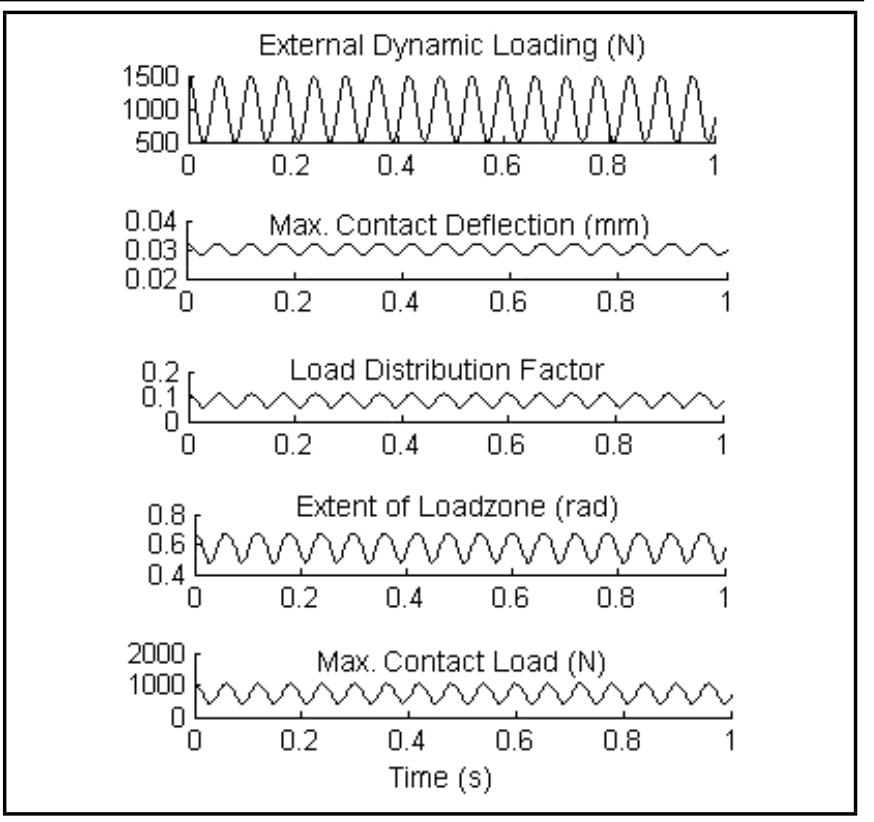

Figure 5. Kinematic and dynamic parameters of bearing for external harmonic load at $1000 \mathrm{rpm}, W=1000 \mathrm{~N}$ and $A=500 \mathrm{~N}$.

Frequency spectra of the demodulated signals have been obtained to identify the important frequency components. Frequency spectra for bearings with $500 \mu \mathrm{m}$ defect on the roller and running at $1000 \mathrm{rpm}$ under a load of $W=1000 \mathrm{~N}$ and $A=0 ; 0.5 W(500 \mathrm{~N}) ; W(1000 \mathrm{~N})$; and $2 W(2000 \mathrm{~N})$ are shown in Figs. 7(a) to 7(d). The representations of symbols in these figures are as follows.

For a bearing with static radial load and no harmonic load, the spectrum has significant harmonic coefficients only at the roller defect frequency and its harmonics, $n f_{r d}$ with sidebands through integer harmonics of cage frequencies, $\pm m f_{c}$ (Fig. 7(a)). This is because the magnitude variation and periodicity of load distribution, $P_{\psi}$ will be uniform while the extent and location of load zone, $\pm \psi_{l}$ remain constant in the case of static loading. The results coincide with the theoretical and experimental observations made by earlier researchers. ${ }^{15,21}$

However, with the introduction of harmonic load, the spectra show additional harmonic coefficients or sidebands at $\pm f_{s}$ about $\left(n f_{r d} \pm m f_{c}\right)$ as shown in Figs. 7(b) to 7(d). The new sidebands may be due to the modulation of load zone by loading frequency, $f_{s}$. It is also observed from these figures that the amplitudes of the additional sidebands significantly increase with an increase in the value of $A$. The rise in amplitudes of the additional sidebands relative to $n f_{r d}$ and $\left(n f_{r d} \pm m f_{c}\right)$ are considerable when amplitude of the dynamic component of external load is higher than that of its static component.

\subsection{External Random Loading}

The external dynamic load on bearing has been considered to be a combination of static load, $W$ and random load, $R(t)$ with known values of mean $\mu$ and standard deviation $\sigma$. The external dynamic load can thus be expressed as

$$
F_{r}(t)=W+R(t)
$$

The variations in bearing kinematic and dynamic parameters presented in Fig. 8 for an external dynamic load with a static component of $1000 \mathrm{~N}$ and random component with $\mu$ and $\sigma$ equal to 0 and $1000 \mathrm{~N}$, respectively. It is clear that all these parameters have random variations with respect to time 


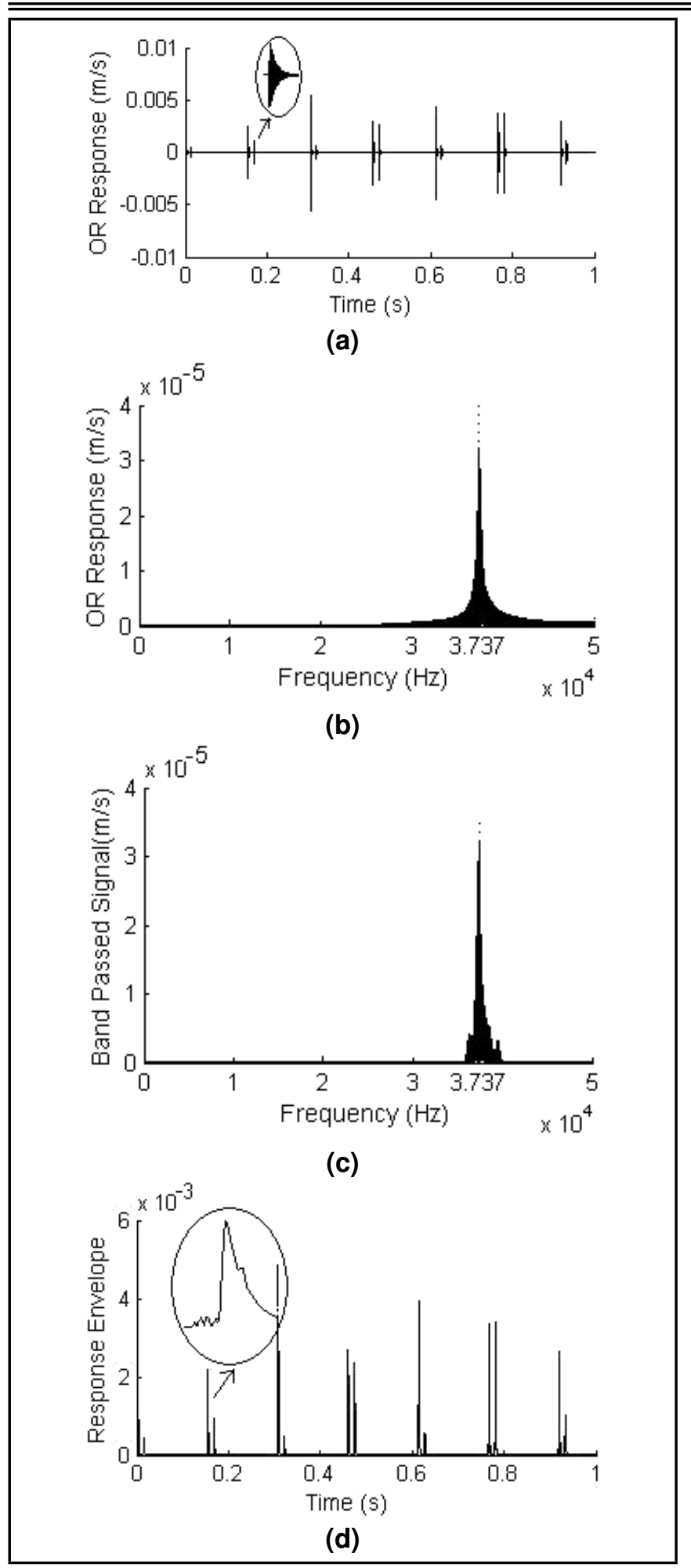

Figure 6. (a) Response signal, (b) spectrum of response signal, (c) spectrum of band pased signal, and (d) envelope of band passed signal.

unlike that of static loading. From Fig. 8 the variations shown at, above, and below the origin represent the variations at the bottom and top of the bearing symmetrical to load line. The response signal processing procedure similar to that for harmonic loading has been applied with an objective to identify defect diagnosis frequencies.

Frequency spectra for a bearing with a 500 micron defect on the roller under a load with static component $W=1000 \mathrm{~N}$ and random component with mean $\mu=0$ and different values of standard deviation $\sigma$ are shown in Figs. 9(a) to 9(d). Since a static component of load has been considered, the mean of the random component has been considered to be zero. The values of standard deviation have been varied to assess the effect of dispersion of random loading.

The significant harmonic coefficients can be observed at harmonics of $f_{r d}$ with side bands at integer multiples of $f_{c}$ from Figs. 9(a) to 9(d). Such spectra are clearly indicative of existence of roller defect. The random noise can also be observed with the random loading, and its level is quite low for low values of standard deviation, as in Figs. 9(a) and 9(b), and considerably high for higher values of standard deviation, as in Figs. 9(c) and 9(d). Significant rise in noise level relative to that of harmonic coefficients can be observed for higher values of standard deviation so that quite a few spectral components have also been masked under the noise level as shown in Figs. 9(c) and 9(d).

\section{EXPERIMENTAL VALIDATION}

In this study, experiments have been conducted to obtain the vibration response of a bearing with a defect on a roller when the bearing is subjected to dynamic load. The test rig used for this purpose is shown in Fig. 10(a). Figure 10(b) shows the defective bearing with line defect on rolling element. The test rig consists of a rigid base frame (1) fabricated from steel channel sections. The frame is grounded to a reinforced concrete and cement foundation (12) by means of foundation bolts. A 3 phase, 3 H.P. foot mounting, ABB make AC motor (4) is bolted to the frame. There is a stepped shaft (6) supported on two deep groove ball bearings (7) of SKF make with 6009 designation. These bearings are located in Plummer block housings which are mounted on the frame by means of lock nuts and washers. The shaft which has been extended beyond the right support bearing is having different step diameters ranging from $25 \mathrm{~mm}$ to $45 \mathrm{~mm}$ at steps of $5 \mathrm{~mm}$ so that the test bearings (8) of different diameters can be mounted on them for experimentation. The motor shaft and stepped shaft are connected by a flexible coupling (5) to minimize the driving component vibration. The speed of motor has been regulated in the range of 0 to $2975 \mathrm{rpm}$ by the variac (3), an auto transformer. The variac used is a 3 phase portable type desktop model suitable for operation from a nominal input voltage of $415 \mathrm{~V}, 3$ phase $\mathrm{AC}$ and gives an output of 0 to $470 \mathrm{~V}$. The static and dynamic loading arrangements have been made in the test rig. The static loading arrangement on the bearing is done by connecting a mild steel platform (9) of $30 \mathrm{~cm} \times 35 \mathrm{~cm} \times 0.05 \mathrm{~cm}$ to the pedestal of test bearing (8) so that loads can be placed on the platform to load the bearing statically. A disc with an unbalanced mass (10) is attached to the shaft to subject the bearing to dynamic loading. A VIB 6.142 DEX accelerometer with magnetic holder (11) is placed above the test bearing to capture the vibration of bearing in the vertical direction. The vibration velocity response of defective bearing has been captured with the help of VIB 6.142 DEX accelerometer and VIBXpert, a data signal analyser. The VIBXpert is a device that collects the response of a vibrating surface and stores vibration signal data into the instrument. The device attached with the probe can be held magnetically on the vibrating surface and feeds the response into the instrument. The probe has been mounted on top of the test bearing housing so that vibration can be measured in the vertical direction. Finally, the data set representing the vibration signal in time domain has been retrieved with the help of OMINITREND PC software and processed further in MATLAB. 


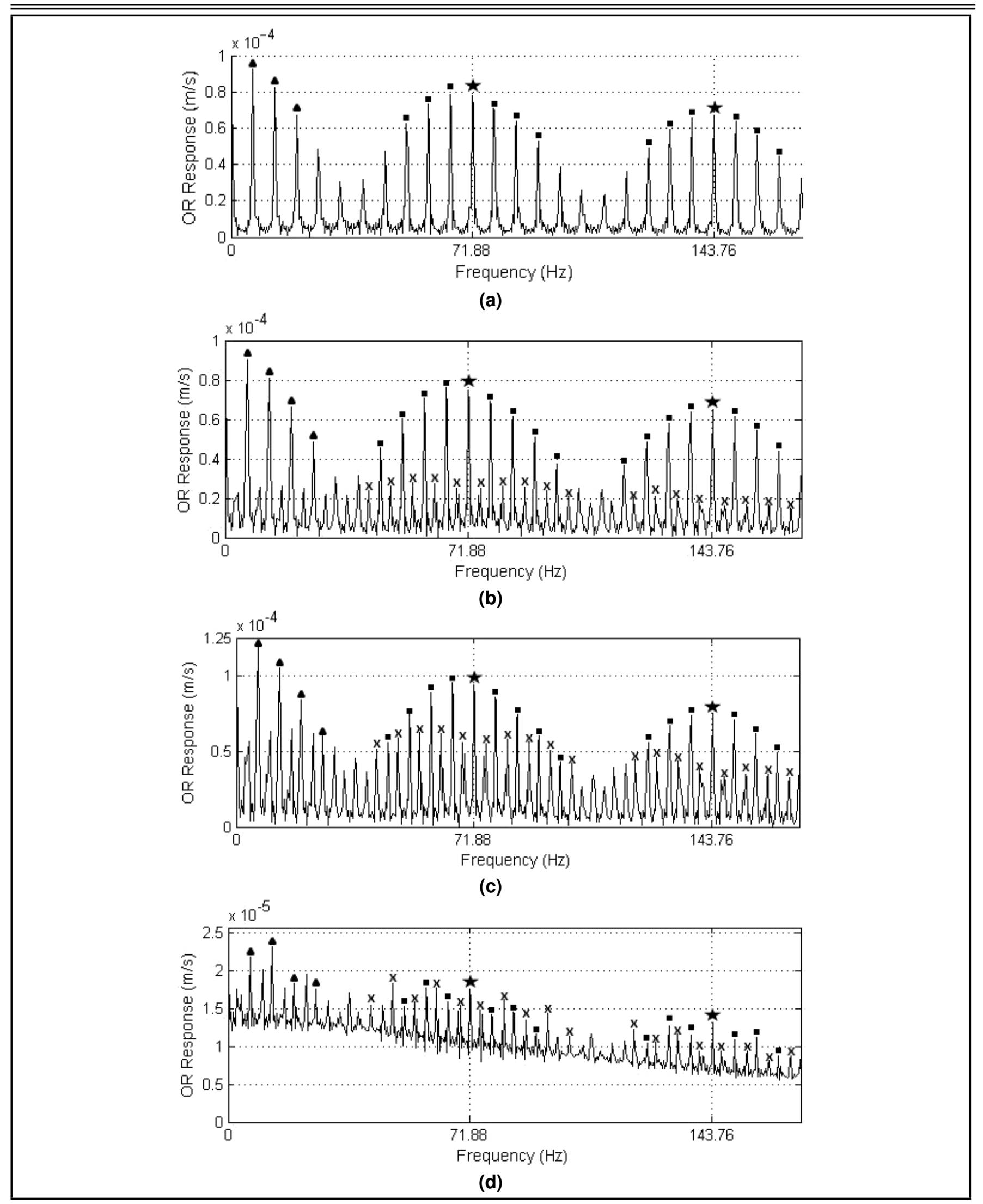

Figure 7. Frequency spectra for NJ 204 bearing with roller defect at $1000 \mathrm{rpm}, W=1000 \mathrm{~N}$; (a) $A=0$; (b) $A=0.5 W ;$ (c) $A=W ;($ d) $A=2 W$. $\boldsymbol{\Delta}: m \times f_{c} ; \star: n \times f_{r d} ; \mathbf{\square}:\left(n \times f_{r d} \pm m \times f_{c}\right) ; \times:\left(n \times f_{r d} \pm p \times f_{c}\right) \pm f_{s} .(m=n=1,2,3 \ldots$ and $p=0,1,2,3 \ldots)$.

The velocity response of bearing with defect on cylindrical rolling element and running at $2975 \mathrm{rpm}$ has been captured in time domain. The captured raw signal has been processed according to the procedure discussed and presented in section 2.4 and Fig. 6, respectively, for diagnosis of defect. The spec- trum of processed response signal obtained for harmonic load in addition to static load has been presented in Fig. 11. In this spectrum, the spectral coefficients are presented on a logarithmic scale because of a greater value of shaft frequency coefficient compared to other significant frequency coefficients. The 


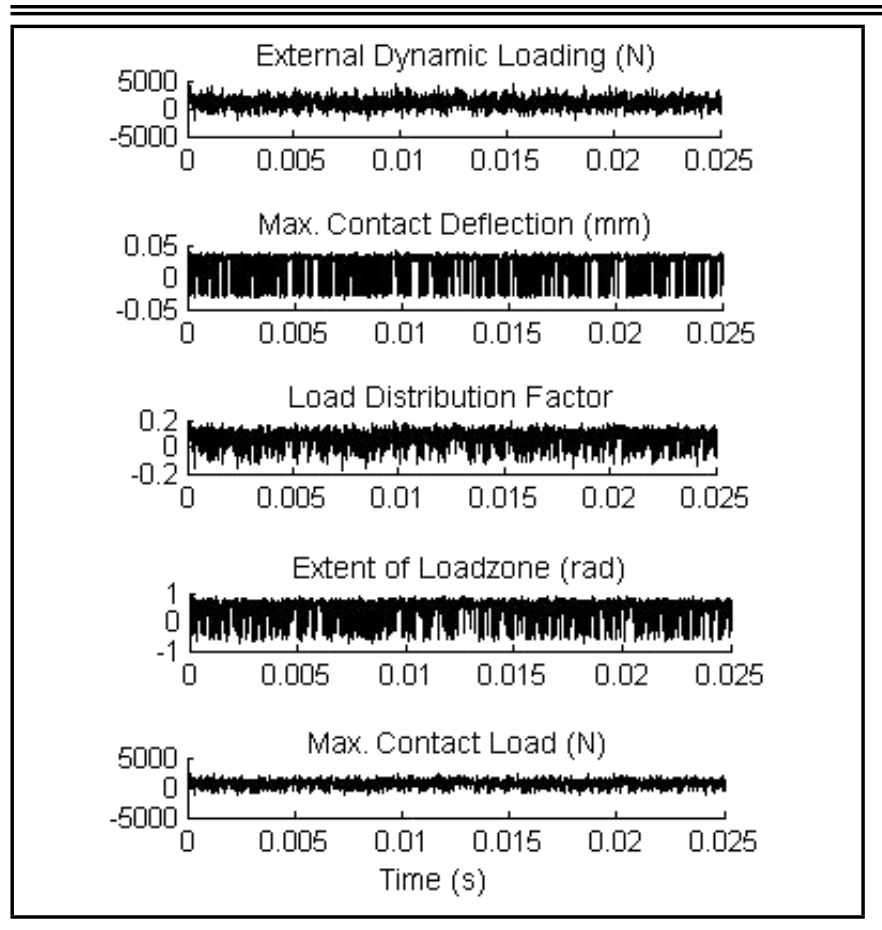

Figure 8. Kinematic and dynamic parameters of bearing for external random load at $1000 \mathrm{rpm}, W=1000 \mathrm{~N}$, and $\mu=0$ and $\sigma=1000 \mathrm{~N}$.

cage, shaft, and rolling element characteristic defect frequencies corresponding to the bearing running speed are $19.9 \mathrm{~Hz}$, $49.6 \mathrm{~Hz}$ and $244.1 \mathrm{~Hz}$, respectively. From these figures the new spectral component labelled by the cross mark can be observed. The new spectral component appears as symmetrically spaced side bands through shaft frequency about each significant frequency component of static loading. The significant frequency component and its new sidebands have been shown in the same colour for easy identification. It can be observed that the pattern of experimental response spectrum in the respect of significant frequency components is the same as that of theoretical.

\section{CONCLUSIONS}

In the present study, an investigation into defect-induced vibration response of a rolling element bearing with a localized defect on cylindrical roller under the influence of external dynamic loading has been analysed. The external load considered in the present study consists of a static component and a dynamic component. The dynamic component has been considered to be either harmonic or random in nature.

Different bearing kinematic and dynamic parameters, viz., maximum deflection, the load distribution factor, the extent of load zone and maximum contact load along load line have been determined for the external loadings considered in the study. These parameters have been found to vary significantly with respect to time for both harmonic as well as random loading.

The location of defect at every instant with respect to the dynamic load zone is investigated and internal load over defect has been calculated with the help of various instantaneous bearing kinematic and dynamic parameters. The strength and periodicity of excitations causing the bearing to vibrate have been investigated under the dynamic load on defect and load zone at every instant.

For harmonic loading, the results for the roller defect show additional spectral components through shaft frequency about each significant spectral component resulted due to static loading. The experimental investigation confirms the presence of these additional components in the spectra. The magnitude of additional components increase with an increase in amplitude of harmonic loading, whereas the sidebands at twice the cage frequency are found to rise significantly when the amplitude of the harmonic component of the external load is greater than its static component.

For random loading, the noise level tends to rise with an increase in dispersion of load represented by standard deviation. The increased noise level for higher values of standard deviation has masked some significant spectral components in the case of roller defect.

\section{REFERENCES}

1 Tandon, N. and Choudhury, A. A review of vibration and acoustic measurement methods for the detection of defects in rolling element bearings, Tribology International, 32 (8), 469-480, (1999). https://dx.doi.org/10.1016/S0301679X(99)00077-8

2 Taylor J. I. Identification of bearing defects by spectral analysis, Journal of Mechanical Design, 102 (2), 199-204, (1980). https://dx.doi.org/10.1115/1.3254730

3 McFadden, P. D. and Smith, J. D. Model for the vibration produced by a single point defect in a rolling element bearing, Journal of Sound and Vibration, 96 (1), 69-82, (1984). https://dx.doi.org/10.1016/0022-460x(84)90595-9

4 Antoni, J. Cyclic spectral analysis of rolling element bearing signals: facts and fictions, Journal of Sound and Vibration, 304 (3-5), 497-529, (2007). https://dx.doi.org/10.1016/j.jsv.2007.02.029

5 Randall, R. B. and Antoni, J. Rolling element bearing diagnostics-a tutorial, Mechanical Systems and Signal Processing, 25 (2), 485-520, (2011). https://dx.doi.org/10.1016/j.ymssp.2010.07.017

6 Holm-Hansen, B. T., Gao, R. X., and Zhang, L. Customized wavelet for bearing defect detection, Journal of Dynamic Systems, Measurement and Control, 126 (4), 740745, (2004). https://dx.doi.org/10.1115/1.1850534

7 Yan R., Gao R. X., and Chen X. Wavelets for fault diagnosis of rotary machines: a review with applications, Signal Processing, 96 (A), 1-15, (2014). https://dx.doi.org/10.1016/j.sigpro.2013.04.015

8 Kiral, Z. and Karagulle, H. Vibration analysis of rolling element bearings with various defects under the action of an unbalanced force, Mechanical Systems and Signal Processing, 20 (8), 1967-1991, (2006). https://dx.doi.org/10.1016/j.ymssp.2005.05.001

9 Sassi, S., Badri, B., and Thomas, M. A numerical model to predict damaged bearing vibrations, Journal of Vibration Control, 13 (11), 1603-1628, (2007). https://dx.doi.org/10.1177/1077546307080040

10 Cong, F., Chen, J., Dong, G., and Pecht. M. Vibration model of rolling element bearings in a rotor-bearing system for fault diagnosis, Journal of Sound and Vibration, 332 (8), 2081-2097, (2013). https://dx.doi.org/10.1016/j.jsv.2012.11.029 


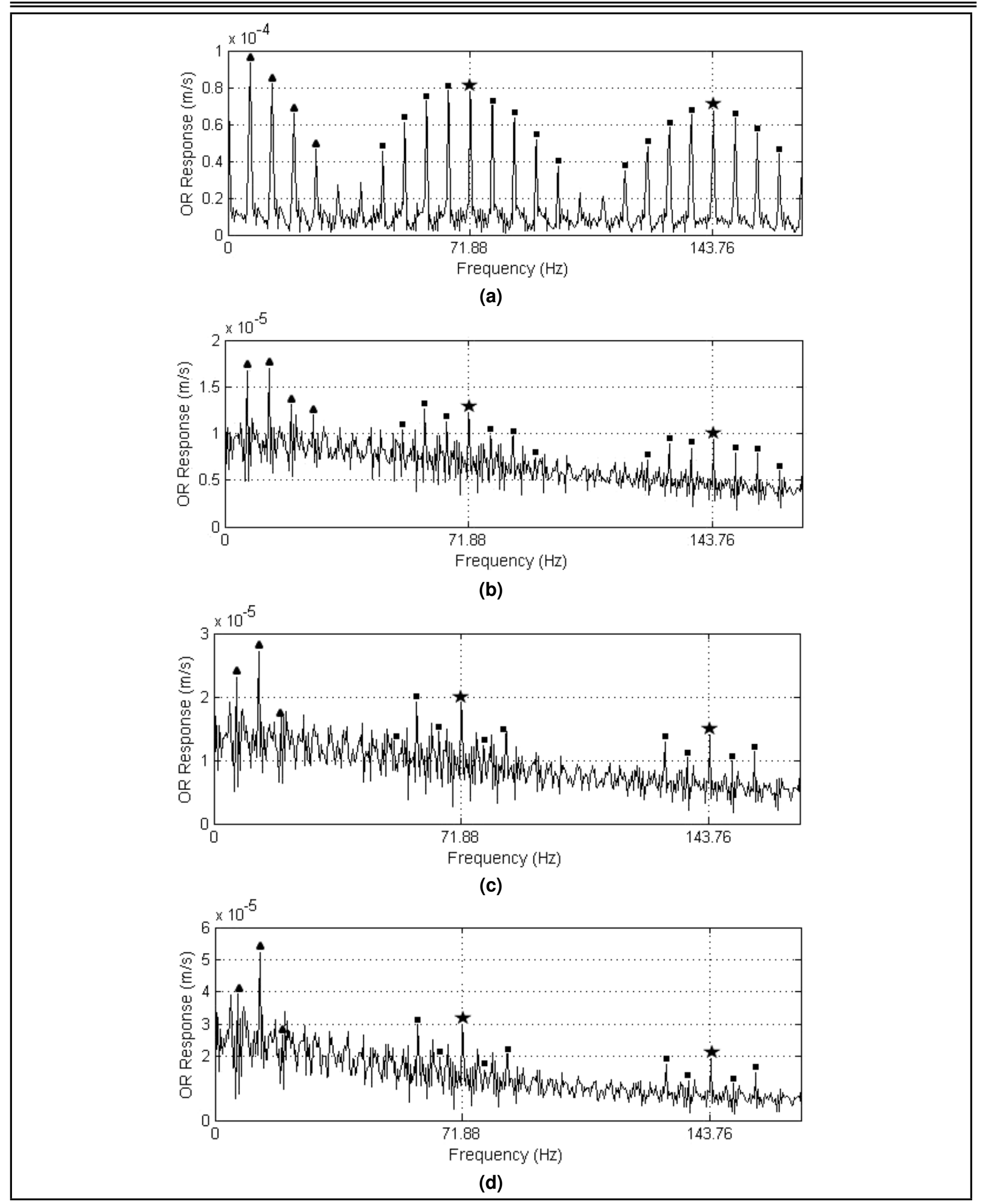

Figure 9. Frequency spectra for NJ 204 bearing with $500 \mu \mathrm{m}$ roller defect at $1000 \mathrm{rpm}, W=1000 \mathrm{~N}, \mu=0$; (a) $\sigma=250$; (b) $\sigma=1000$; (c) $\sigma=2000$; (d) $\sigma=3000$. $\boldsymbol{\Delta}: m \times f_{c} ; \star: n \times f_{r d} ; \mathbf{\square}:\left(n \times f_{r d} \pm m \times f_{c}\right)$.

11 Govardhan, T., Choudhury, A., and Paliwal, D. Vibration analysis of a rolling element bearing with localized defect under dynamic radial load, Journal of Vibration Engineering and Technologies, 5 (2), 165-175, (2017)

12 Govardhan, T., Choudhury,
D. Load distribution in a rolling element bearing under dynamic radial load, Applied Mechanics and Materials, 592-594, 1099-1103, (2014). https://dx.doi.org/10.4028/www.scientific.net/amm.592594.1099 


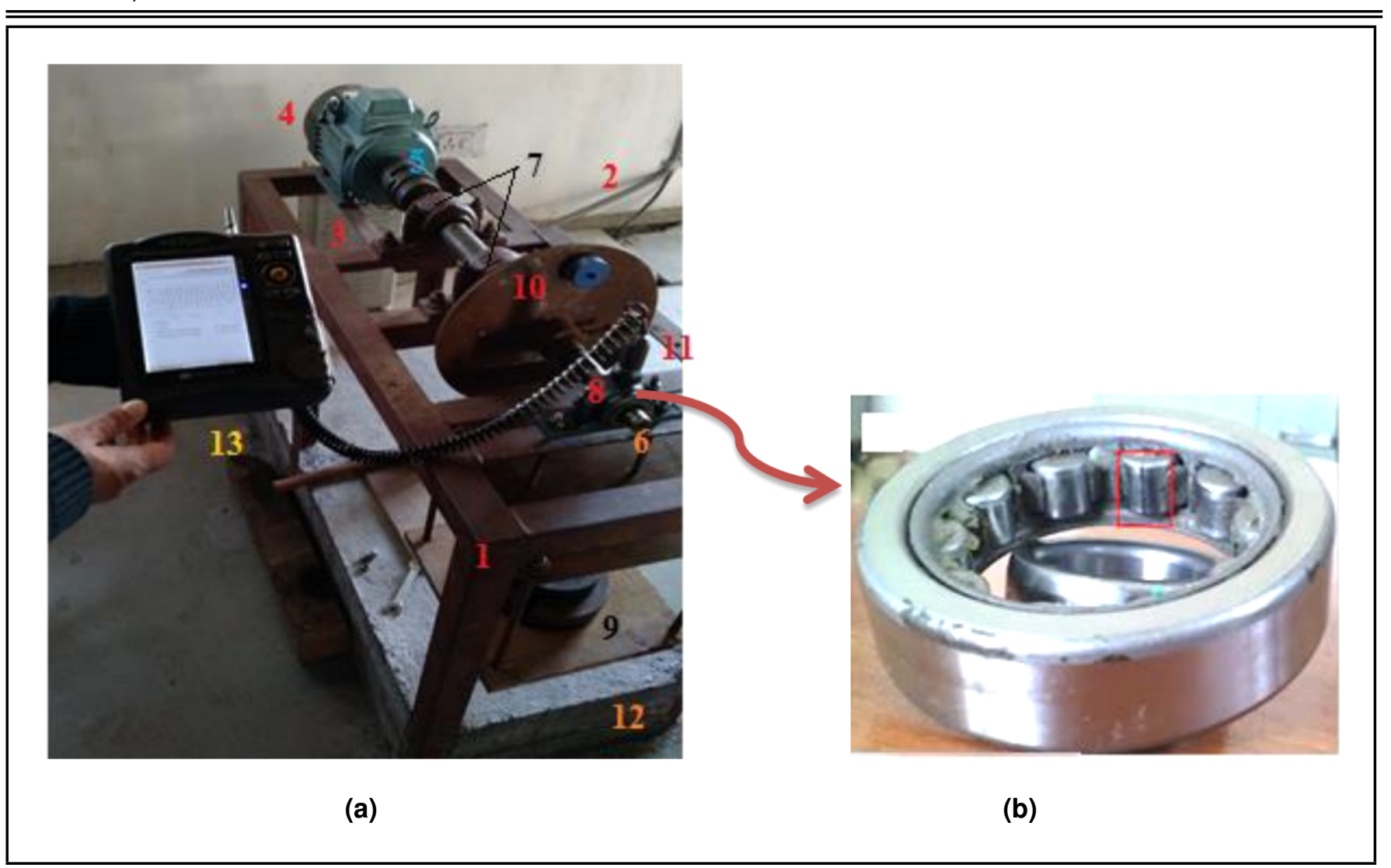

Figure 10. (a) Photographic view of rotor-bearing test set up and (b) defective bearing.

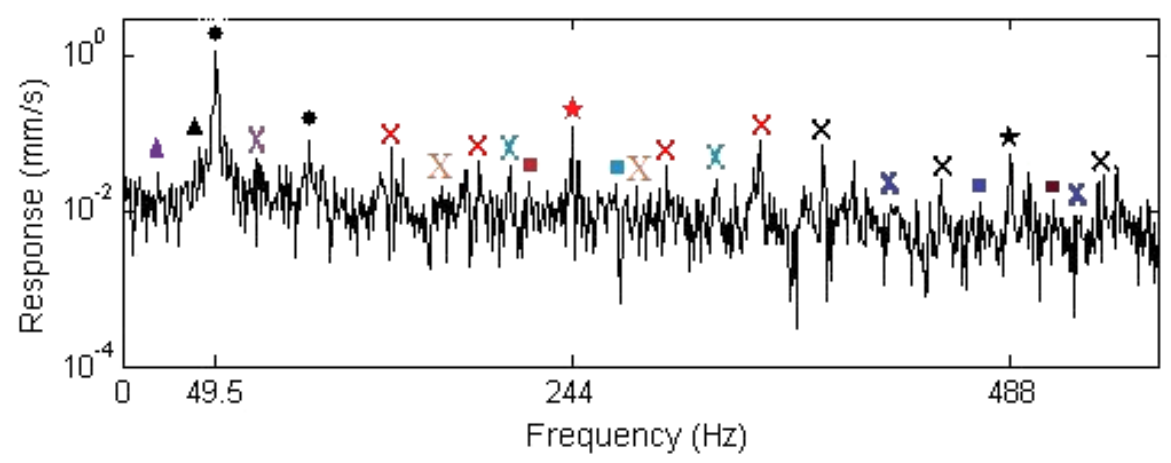

Figure 11. Experimental response spectra of bearing with defect on rolling element and running at a speed of 2975 rpm under dynamic loading conditions $W=200 \mathrm{~N}$ and $A=W . \mathbf{\Delta}: m \times f_{c} ; \star: n \times f_{r d} ; \mathbf{\square}:\left(n \times f_{r d} \pm m \times f_{c}\right) ; \times:\left(n \times f_{r d} \pm p \times f_{c}\right) \pm f_{s} ;: m \times f_{s}$.

13 Govardhan, T., Choudhury, A., and Paliwal, D. An investigation into defect induced excitations in rolling element bearings under dynamic radial load, Proc. 2015 International Conference on Industrial Instrumentation and Control, Pune, India, 217-222, (2015). https://dx.doi.org/10.1109/IIC.2015.7150741

14 Harris T. A. Rolling Bearing Analysis, John Wiley, Chichester, (1984).

15 Choudhury, A. and Tandon, N. Vibration response of rolling element bearings in a rotor-bearing system to a local defect under radial load, Journal of Tribology, 128 (2), 252-261, (2006). https://dx.doi.org/10.1115/1.2164467

16 Eschmann, P. Ball and Roller Bearings-Theory, Design and Application, John Wiley, New York, (1985).
17 Weaver, Jr. W., Timoshenko, S. P., and Young, D. H. Vibration problems in engineering, John Willey, New York, (1990).

18 Adams, M. L. Rotating machinery vibrations: from analysis to troubleshooting, CRC Press, (2000).

19 Hamrock, B. J. Fundamentals of fluid film lubrication, Singapore, McGraw-Hill, (1994).

20 Hamrock, B. J. and Dowson, D. Ball bearing lubrication: the elastohydrodynamics of elliptical contacts, Wiley, New York, (1981).

21 Tandon, N. and Choudhury, A. An analytical model for the prediction of the vibration response of rolling element bearings due to a localized defect, Journal of Sound and Vibration, 205 (3), 275-292, (1997). https://dx.doi.org/10.1006/jsvi.1997.1031 